Chinese Science Bulletin 2006 Vol. 51 No. 13 1648-1651

DOI: $10.1007 / \mathrm{s} 11434-006-2020-5$

\section{Analyses of nitrobenzene, benzene and aniline in envi- ronmental water samples by headspace solid phase micro- extraction coupled with gas chromatography-mass spectrometry}

\author{
CHEN Ming ${ }^{1,2}$, YIN Yongguang ${ }^{1}$, TAI Chao ${ }^{1}$, \\ ZHANG Qinghua ${ }^{1}$, LIU Jiyan ${ }^{1}$, HU Jingtian ${ }^{2}$ \\ \& JIANG Guibin ${ }^{1}$
}

1. State Key Laboratory of Environmental Chemistry and Ecotoxicology, Research Center for Eco-environmental Sciences, Chinese Academy of Sciences, Beijing 100085, China;

2. School of Chemistry and Chemical Engineering, Shandong University, Ji'nan 250100, China

Correspondence should be addressed to Jiang Guibin (email: gbjiang @rcees.ac.cn)

Received January 23, 2006; accepted April 24, 2006

\begin{abstract}
A headspace solid phase microextraction coupled with gas chromatography mass spectrometry (HS-SPME/GC-MS) method was established for analyzing nitrobenzene, benzene and aniline in environmental water samples simultaneously. Factors affecting extraction efficiency (SPME fiber selection, acidity, temperature, salt addition, extraction time, headspace, etc) were verified. Under optimal extraction conditions, the detection limits are $0.50,0.11$ and $1.00 \mu \mathrm{g} / \mathrm{L}$ for nitrobenzene, benzene and aniline, respectively. The results indicate that this method is capable of making sensitive and accuracy analyses on nitrobenzene, benzene and aniline in environmental water samples.
\end{abstract}

Keywords: HS-SPME, GC-MS, nitrobenzene, benzene, aniline.

Nitrobenzene, benzene and aniline are widely used in chemical industries as solvents and reaction intermediates. Their discharge or leakage to the environment will result in serious environmental pollution, so it is very important to propose a fast and accurate analytical method to assess pollution levels and establish corre- sponding measures in emergencies. The liquid-liquid extraction coupled with gas chromatography (LLE/GC) technique was the traditional analytical method ${ }^{1), 2)}$. LLE was laborious, time-consuming, expensive and used toxic organic solvents. Compared with LLE, solid phase microextraction (SPME) offers significant advantages due to its simplicity, short analysis time, easiness of operation and being free of organic solvent. SPME is a new technology of sample preparation, first described in the early $1990 \mathrm{~s}^{[1-4]}$. It involves the adsorption of analytes to a fused silica fiber coated with suitable stationary phases and their subsequent desorption immediately before chromatographic analysis. The analytes can be adsorbed on the fiber by immersing it in the samples or by exposing it on the sample headspace (HS-SPME). There has been a rapid growth in the number of applications of SPME in environmental samples ${ }^{[5,6]}$.

The aim of this work is to optimize the SPME conditions and establish a method for rapid determination of nitrobenzene, benzene and aniline in environmental water samples.

\section{Experiment section}

\subsection{Reagents and instrumentation}

Benzene (99.5\% pure), nitrobenzene (99.8\% pure) and aniline were obtained from Beijing Chemical Reagents Company. Aniline was purified and conserved in dark. All stock solutions were prepared in pure water at the concentration of $100 \mathrm{mg} / \mathrm{L}$, respectively. Mixed standards containing benzene $110 \mu \mathrm{g} / \mathrm{L}$, nitrobenzene $3.6 \mathrm{mg} / \mathrm{L}$ and aniline $140 \mu \mathrm{g} / \mathrm{L}$ were also prepared. A Milli-Q water purifying system was used to prepare aqueous solutions.

The manual holder and microextraction fiber, PDMS (polydimethylsioxane, $100 \mu \mathrm{m}$ ) and PA (polyacrylate, $85 \mu \mathrm{m})$, are from Supelco Inc (Bellefonte, PA, USA). Magnetic stirrer is from Huifeng Electronic Instruments Company.

An Agilent 6890N GC equipped with Agilent 5973N MSD fitted with a DB-5 capillary column $(30 \mathrm{~m} \times 0.25$ $\mathrm{mm}, 25 \mu \mathrm{m}$ ) was used for analysis. Helium was used as the carrier gas at a flow rate of $1 \mathrm{~mL} \mathrm{~min}^{-1}$. Two oven temperature programs were as follows, program 1: $35^{\circ} \mathrm{C}$ held for $3 \mathrm{~min}, 6^{\circ} \mathrm{C} \mathrm{min}^{-1}$ up to $60^{\circ} \mathrm{C}, 10^{\circ} \mathrm{C} \min ^{-1}$

1) GB11890, Water quality-determination of benzene and its analogies-gas chromatographic method.

2) GB13194, Water quality-determination of nitrobenzene, mononitrotoluene (MNT), nitrochlorobenzene, and dinitrotoluene (ONT)-gas chromatography. 
up to $110^{\circ} \mathrm{C}, 110^{\circ} \mathrm{C}$ held for $1 \mathrm{~min} ; 30^{\circ} \mathrm{C} \mathrm{min}^{-1}$ up to $250^{\circ} \mathrm{C}$; program $2: 50^{\circ} \mathrm{C}$ held for $1 \mathrm{~min}, 6^{\circ} \mathrm{C} \mathrm{min}-1$ up to $110^{\circ} \mathrm{C}, 30^{\circ} \mathrm{C} \min ^{-1}$ up to $270^{\circ} \mathrm{C}, 270^{\circ} \mathrm{C}$ held for $1 \mathrm{~min}$. Purge time: $0.1 \mathrm{~min}$ in program $1,0.75 \mathrm{~min}$ in program 2. Injector mode and temperature: splitless, $250^{\circ} \mathrm{C}$. Purge flow: $30 \mathrm{~mL} \mathrm{~min}^{-1}$. The mass detector was chosen to use selective ion monitoring mode (SIM). The recording ions were: benzene, $m / z 78,77,63$; aniline, $m / z$ 93, 66, 65; nitrobenzene, $m / z$ 123, 77, 65 .

\subsection{Sample collection}

All water samples were collected along Songhua River in Jilin, Songyuan and Zhaoyuan in Jilin Province, and Harbin, Jiamusi and Tongjiang in Heilongjiang Province and stored at $-20^{\circ} \mathrm{C}$.

\subsection{Experiment methods}

A $25 \mathrm{~mL}$ sample solution was placed into $50 \mathrm{~mL}$ vial, and then the vial was pressurized with parafilm. SPME fiber was exposed to the headspace of the vial to enrich analyte, and then thermally desorbed in heated GC injector for analysis. The extraction process was under stirring. $8 \mathrm{~g}$ of $\mathrm{NaCl}$ and $0.1 \mathrm{~g}$ of $\mathrm{NaHCO}_{3}$ were added to regulate the salinity and acidity. Extraction time is 10 min and optimal temperature is $25^{\circ} \mathrm{C}$. All the SPME conditions were the same as described above (the extraction instruments are shown in Fig. 1).

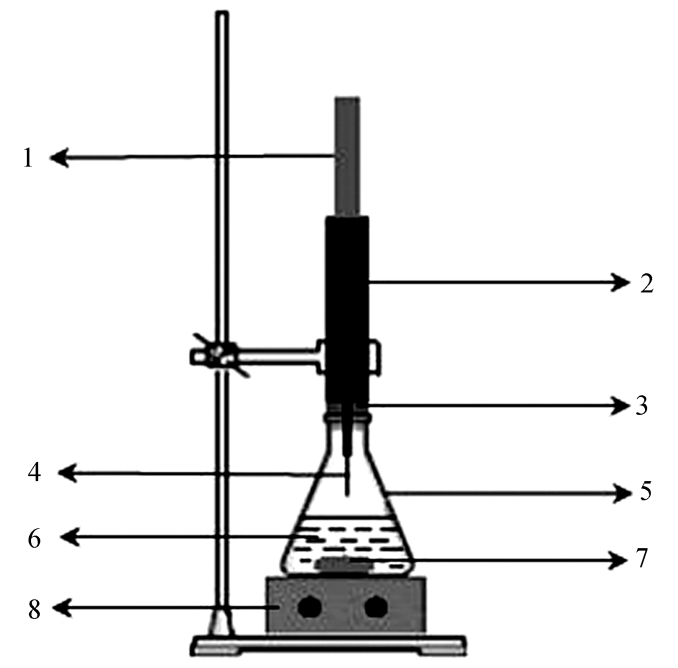

Fig. 1. Extraction instruments. 1, Holder; 2, needle guide; 3, rubber spill; 4, extraction fiber; 5 , vial; 6 , solvent; 7, magneton; 8 , magnetic stirrer.

\section{Results and discussion}

\subsection{Selection of purge time}

The boiling point of benzene is lower than nitroben- zene and aniline, and the retention time of benzene is short, so purge time has great effect on its detection. The peak shape broadened severely when purge time increased, and when the purge time was $0.75 \mathrm{~min}$, benzene could not be detected normally; but when purge time was reduced, peak shape of benzene was slightly amended while detection sensitivity of aniline decreased much. So nitrobenzene and aniline were detected together with $0.75 \mathrm{~min}$ of purge time; benzene was detected with $0.1 \mathrm{~min}$ of purge time. As shown in Fig. 2, the three compounds could be well separated.

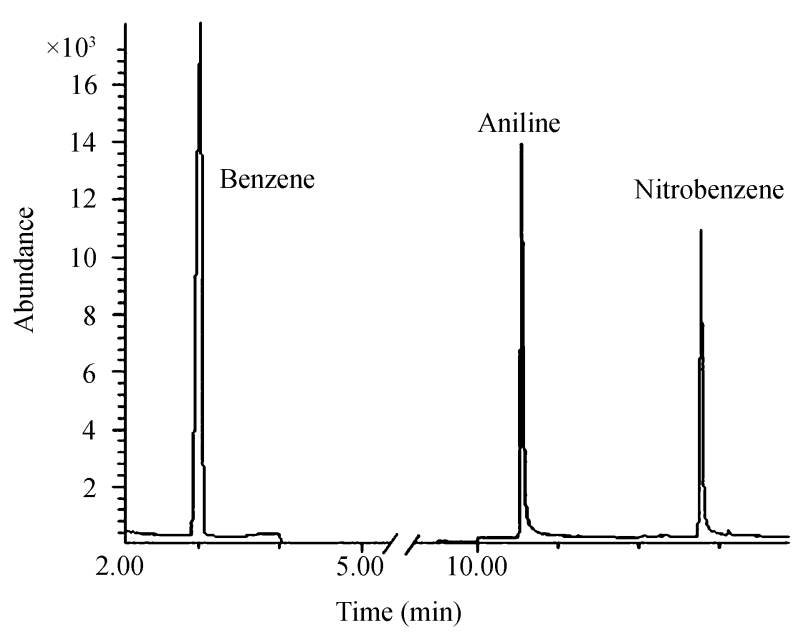

Fig. 2. Chromatogram of benzene, aniline and nitrobenzene (benzene: $11 \mu \mathrm{g} / \mathrm{L}$; aniline: $360 \mu \mathrm{g} / \mathrm{L}$; nitrobenzene: $14 \mu \mathrm{g} / \mathrm{L}$ ) analyzed by temperature program 1 with $0.1 \mathrm{~min}$ of purge time.

\subsection{Selection of SPME fiber}

Now polydimethylsioxane (PDMS) and polyacrylate (PA) fibers are widely used as commercial extraction fibers. A comparison of extraction efficiency was made between them. When PA fiber was used, extraction efficiency of benzene and nitrobenzene decreased while that of aniline increased. When PDMS fiber was used, the extraction process could reach equilibrium in 10 min with high extraction efficiency. So PDMS fiber was used in further experiments.

\subsection{Selection of extraction time}

Because SPME is an equilibrium method, time to reach relatively extraction balance is important. As shown in Fig. 3, the peak area of all the three analytes increased corresponding to the increase of extraction time, and had basically no change after $10 \mathrm{~min} .10 \mathrm{~min}$ was the optimal time. 


\section{BRIEF COMMUNICATION}

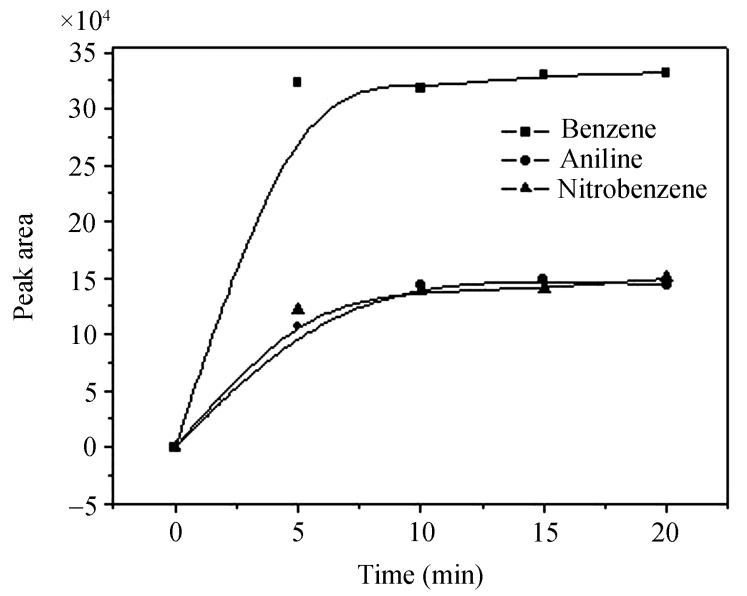

Fig. 3. Effect of extraction time on extraction efficienty.

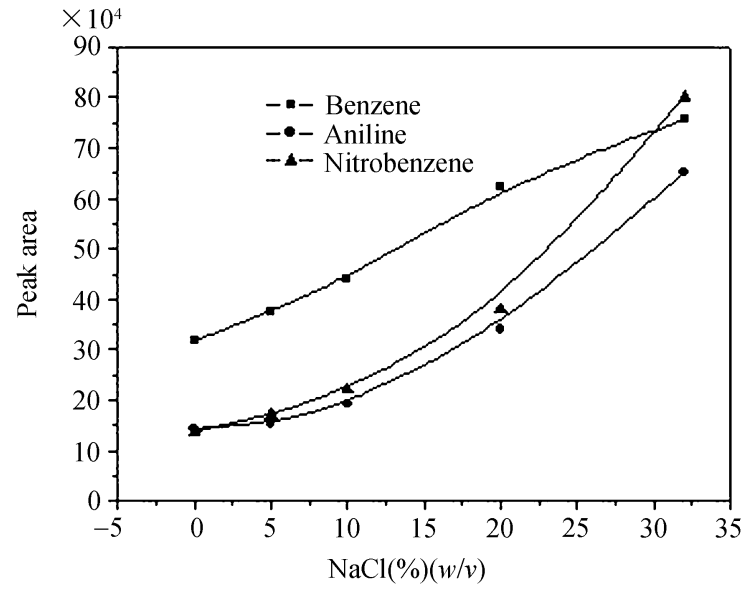

Fig. 4. Extraction effect of salinity.

Table 1 Calibration curves and detection limits

\begin{tabular}{lcccc}
\hline \multicolumn{1}{c}{ Analytes } & Linear equation & Linear range $(\mu \mathrm{g} / \mathrm{L})$ & $R^{2}$ & Detection limits $(\mu \mathrm{g} / \mathrm{L})$ \\
\hline Benzene ${ }^{\mathrm{a})}$ & $Y=37399+4560 X$ & $1.1-440$ & 0.994 & 0.11 \\
Aniline & $Y=31411+2057 X$ & $4.4-704$ & 0.999 & 1.00 \\
Nitrobenzene & $Y=5209+2838 X$ & $2.4-960$ & 0.998 & 0.50 \\
\hline
\end{tabular}

a) $0.1 \mathrm{~min}$ of purge time detected alone.

Table 2 Determination of an environmental water sample and a spiked water sample

\begin{tabular}{lcccc}
\hline \multicolumn{1}{c}{ Analytes } & Detected values $(\mu \mathrm{g} / \mathrm{L})$ & Spiked $(\mu \mathrm{g} / \mathrm{L})$ & Found values $(\mu \mathrm{g} / \mathrm{L})$ & Recovery $(\%)$ \\
\hline Benzene $^{\text {a) }}$ & N.D. & 6.5 & 6.8 & 104.6 \\
Aniline & N.D. & 69.3 & 71.5 & 103.2 \\
Nitrobenzene & 5.9 & 7.9 & 12.4 & 82.3 \\
\hline
\end{tabular}

a) 0.1 min of purge time detected alone.

\subsection{Effect of salinity}

Extraction efficiency could be increased by the addition of $\mathrm{NaCl}$. With the effect of salting out, solubility of organic compounds was reduced, and more volatile compounds partitioned into the headspace of the sample solution. Fig. 4 shows the effect of salt addition. Extraction efficiency of all the three analytes increased obviously. So the optimal condition was $8 \mathrm{~g}$ of $\mathrm{NaCl}$ added into $25 \mathrm{~mL}$ sample solution.

\subsection{Effect of extraction temperature}

When other factors were held constant, extraction efficiency of benzene and nitrobenzene decreased and that of aniline increased with the increase of temperature. For convenience of control of temperature, the optimal temperature was $25^{\circ} \mathrm{C}$.

\subsection{Effect of $p H$}

It was shown in experiments that $\mathrm{pH}$ had little effect on benzene and nitrobenzene extraction, but had great effect on aniline due to its ionization in acid condition.
So $\mathrm{pH} 8.4$ has been chosen as the optimal condition by adding $0.1 \mathrm{~g}$ of $\mathrm{NaHCO}_{3}$ into 25 sample solution.

\subsection{Calibration curves and detection limits}

All the optimal calibration curves, liner ranges and correlations are listed in Table 1 (When the concentration of benzene was lower than $1.1 \mu \mathrm{g} / \mathrm{L}$, linear relation did not exist although there was high peak).

\subsection{Replicate experiments}

After injection of 5 replicate samples, relative standard deviation (RSD) of each analyte was $8.2 \%$ for benzene, $7.0 \%$ for aniline and $4.4 \%$ for nitrobenzene, respectively.

\subsection{Determination of environmental water samples}

Two water samples were analyzed by the method described above, and the results are listed in Table 2. Extraction recovery was calculated by addition of mixed standards to water samples. Fig. 5 shows the chromatography of environmental water sample and 


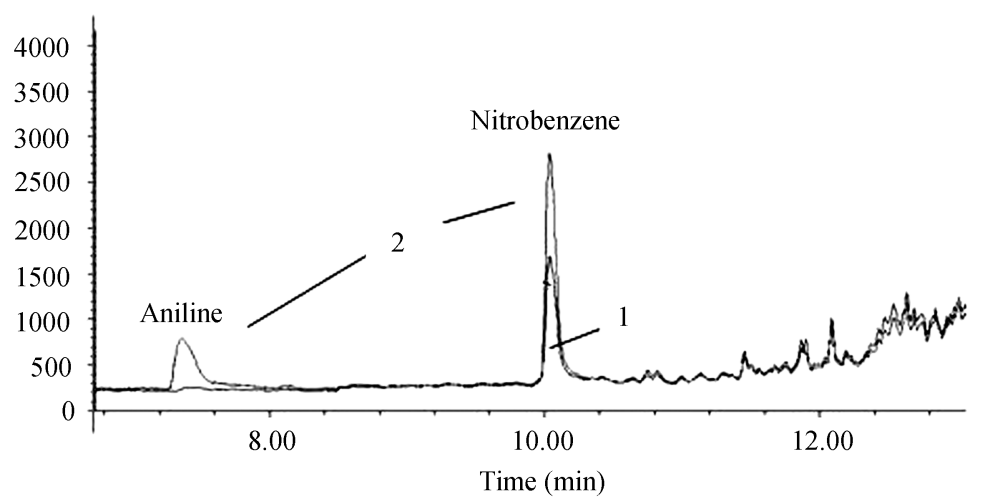

Fig. 5. Chromatography of environmental water sample and spiked water sample. 1, Environmental sample; 2, spiked water sample.

spiked water sample.

\section{Conclusion}

An HS-SPME-GC-MS method was established to detect the nitrobenzene, benzene and aniline simultaneously. Environmental samples could be analyzed directly after HS-SPME, and no excess sample preparation was needed. This method had the characteristics of rapidness, accuracy and easy operation, so it was suitable for routine analyses of nitrobenzene, benzene and aniline in environmental water samples.

Acknowledgements This work was supported by the Chinese Academy of Sciences.

\section{References}

1 Lord H, Pawliszyn J, Evolution of solid-phase microextraction technology. J Chromatogr A, 2000, 885: 153-193

2 Xia X, Leidy R B. Preparation and charaterization of porous silic-coated multifibers for solid-phase microextraction. Anal Chem, 2001, 73: 2041-2047

3 Prosen H, Zupančič-Kralj L. Solid-phase microextraction. Trends in Anal Chem, 1999, 18: 272-282

4 Geppert H. Solid-phase microextraction with the rotation of the microfiber. Anal Chem, 1998, 70: 3981-3982

5 Pawliszyn J, Górecki T, Applications of Solid Phase Microextraction. RSC Chromatography Monographys. Cambridge: Royal Society of Chemistry, 1999, 92-108

6 Mester Z, Lam J, Sturgeon R, et al. Determination of methylmercury by solid-phase microextraction inductively coupled plasma mass spectrometry: A new sample introduction method for volatile metal species. J Anal At Spectrom, 2000, 15: 837-842 